\title{
Unidades de ortogeriatría
}

Rafael Monte Secades

Servicio de Medicina Interna

Hospital Universitario Lucus Augusti. Lugo

Presidente de la Sociedad Gallega de Medicina Interna (SOGAMI)
El aumento progresivo de la esperanza de vida ligada a la mejora de las condiciones higiénicas, socioeconómicas y de la asistencia sanitaria ha conducido a un incremento constante de la población anciana en los últimos años en nuestro país. En este contexto de envejecimiento, la prevalencia de enfermedades crónicas ha aumentado hasta el punto de convertirse en uno de los mayores retos a los que se enfrenta nuestro sistema sanitario, no sólo desde el punto de vista clínico, sino también en su vertiente social, económica y comunitaria. Este cambio demográfico repercute lógicamente en las características de los pacientes hospitalizados, que cada vez con más frecuencia son enfermos ancianos, pluripatológicos y con una elevada complejidad, tendencia que afecta por igual a las áreas médicas y quirúrgicas.

En los servicios quirúrgicos, las mejoras técnicas y en el proceso de cuidados postoperatorios desarrollados en las últimas décadas, permiten realizar intervenciones a pacientes ancianos que hace poco tiempo se habrían descartado por su edad. La complejidad creciente que implica la asistencia sanitaria en este escenario de multimorbilidad, unida a la progresiva superespecialización que se está imponiendo en las diversas áreas de conocimiento y a problemas organizativos que dificultan con frecuencia el seguimiento diario del paciente por el mismo facultativo, ha favorecido un aumento progresivo de la participación de los servicios médicos en la atención de pacientes quirúrgicos. El internista es, en atención a su polivalencia y capacidad de enfoque global, el especialista más requerido por los servicios quirúrgicos ${ }^{1}$. En los últimos años se han desarrollado nuevos sistemas de colaboración como el de asistencia compartida, basado en la formación de un equipo multi- disciplinar en el que la responsabilidad y autoridad sobre el paciente hospitalizado son compartidas entre el equipo quirúrgico y el clínico, sin necesidad de interconsulta formal ${ }^{2}$.

La fractura osteoporótica de cadera es una patología aguda que requiere ingreso urgente en un servicio quirúrgico y está ligada el envejecimiento, además de a una elevada prevalencia de enfermedades crónicas subyacentes, por lo que constituye un buen modelo clínico para implementar esta visión transversal en la integración asistencial de cuidados hospitalarios. En este número de Galicia Clínica, Brinquinho $\mathrm{M}$ et al describen la experiencia del servicio de medicina interna del hospital de Vila Nova Gaia/Espinho al frente de una unidad de ortogeriatría ${ }^{3}$. Su enfoque multidisciplinar, basado en una valoración precoz de la comorbilidad, una intervención quirúrgica temprana, la prevención de complicaciones, la optimización del tratamiento pre y postoperatorio, y especialmente en la rehabilitación de la marcha ha asociado unos buenos resultados en cuanto a complicaciones intrahospitalarias y estado funcional al alta. La baja prevalencia de comorbilidad de la serie podría justificar la escasa incidencia de complicaciones en relación con trabajos previos $^{4}$. No obstante, hay evidencias de que estas unidades clínico-quirúrgicas mejoran claramente los resultados en la atención de estos enfermos ${ }^{5}$.

La denominación de estas unidades en la literatura médica ha sido variable, dependiendo de las características del centro hospitalario y del equipo que lidera la tarea: unidad multidisciplinar de fractura de cadera, modelo hospitalista de fractura de cadera, unidad geriátrica de fractura de cadera, etc. En los últimos años parece haberse impuesto el término de unidad de ortogeriatría, estimulado por la actividad creciente de la especialidad de 


\section{"La asistencia sanitaria en general y particularmente la hospitalaria de los próximos años estará basada en la integración de equipos multidisciplinares y en la transversalidad de la atención"}

geriatría en este campo. La nomenclatura empleada no debiera servir de elemento disuasorio para la integración de internistas en estos equipos, cuya competencia en la atención de este tipo de pacientes está más que acreditada, como demuestran el artículo de Brinquinho et al y otras experiencias similares en las que Galicia es pionera $^{6}$ ni tampoco para alentar conflictos entre las diferentes especialidades que pueden aportar sus conocimientos en este escenario. Los especialistas en medicina interna que aborden esta tarea deberán adaptar su formación al manejo de conceptos como el de la fragilidad, la estratificación del riesgo preoperatorio y a aspectos específicos relacionados con la rehabilitación, tanto en su vertiente funcional como social, con humildad y determinación, de la misma manera que se han adaptado en el pasado a otros retos emergentes que han planteado los sucesivos cambios epidemiológicos y demográficos, como el SIDA, las enfermedades autoinmunes, o la cronicidad.

La asistencia sanitaria en general y particularmente la hospitalaria de los próximos años estará basada en la integración de equipos multidisciplinares y en la transversalidad de la atención, en la que todos los recursos disponibles deberán ponerse al servicio del enfermo y no del profesional. En este contexto, es probable que la colaboración entre internistas y el resto de las especialidades hospitalarias constituya la regla y no la excepción, adaptada a las características concretas de cada hospital. En definitiva, el conocimiento no es patrimonio de nadie, y los mejores resultados en salud siempre estarán asociados a la suma de talentos y capacidades.

\section{Bibliografía}

1. Rohatgi N, Loftus P, Grujic O, Cullen M, Hopkins J, Ahuja N. Surgical comanagement by hospitalists improves patient outcomes: a propensity score analysis. Ann Surg. 2016: 264: 275-82

2. Montero Ruiz E, Monte Secades R. Definiciones de la actividad de Medicina Interna fuera de su propio servicio. Rev Clin Esp. 2015; 215: 182-5

3. Brinquinho M, Seabra F, Sarmento G, Veríssimo R, Oliveira A. Casuística de uma unidade de Ortogeriatria. Galicia Clin 2017; 78 (4):116-122

4. Fernández-Moyano A, Fernández-0jeda R, Ruiz-Romero V, García-Benítez B, Palmero-Palmero C, Aparicio-Santos R. Programa de atención integral a pacientes mayores de 65 años con fractura de cadera. Rev Clin Esp. 2014; 214: 17-23

5. Grigoryan KV, Javedan H, Rudolph JL. Orthogeriatric care models and outcomes in hip fracture patients: a systematic review and meta-analysis. J Orthop Trauma. 2014 Mar; 28: 49-55

6. Monte-Secades R, Codesido-Vilar P, Pardo-Sobrino FJ, García-Monasterio El, Portero-Vázquez A, Garcia-Novio M, Fernández-Lamelo F. Vía clínica para pacientes hospitalizados con fractura osteoporótica de cadera. Galicia Clin 2016; 77 (2): 57-66 